\title{
REPENSANDO EL IMPUESTO AL VALOR AGREGADO $^{\circ} \circ$
}

\author{
RETHINKING THE VALUE ADDED TAX \\ Marcelo Garriga*
}

enviado: 06 julio 2020 - aceptado: 23 septiembre 2020

\section{Resumen}

El objetivo de este trabajo es estudiar los efectos de la existencia de tasas reducidas, bienes a tasa cero y exenciones del IVA en Argentina sobre los precios de los bienes (efectos sobre la eficiencia económica), el impacto distributivo y la eficiencia recaudatoria. El impuesto al valor agregado se ha constituido a lo largo del tiempo en un tributo clave de la estructura impositiva argentina, con una recaudación de $7.36 \%$ del PBI en el año 2018 y una participación en el total de recursos tributarios del $32.6 \%$. La eficiencia "C" es del 47\%, lo que muestra que el potencial recaudatorio del IVA es importante y cualquier posible reforma tributaria debería poner el foco en la mejora del diseño e implementación de este impuesto.

Palabras clave: eficiencia, impuesto al valor agregado, política fiscal. Código JEL: H20, E62.

\begin{abstract}
The aim of this paper is to study the effects of the existence of reduced rates, zero-rate goods and VAT exemptions in Argentina on the prices of goods (effects on economic efficiency), the distributive impact and collection efficiency. The value

Versiones anteriores de este trabajo fueron publicadas como documento de trabajo en la FCE-UNLP y en las Jornadas Internacionales de Finanzas Públicas de la UNC.

* Garriga, M. (2021). Repensando el impuesto al valor agregado. Estudios económicos, 38(76), 197-222.

* Centro de Estudios de Finanzas Públicas, Facultad de Ciencias Económicas, Universidad Nacional de La Plata, Argentina. Correo electrónico: marcelogarriga@yahoo.com.ar. ORCID: https://orcid. org/0000-0002-9297-9740
\end{abstract}


added tax has become over time a key tribute to the Argentine tax structure, with a collection of $7.6 \%$ of GDP in 2018 and a share in total tax resources of $32.6 \%$. Efficiency " $\mathrm{C}$ " is $47 \%$, which shows that the potential for VAT collection is important and any possible tax reform should focus on improving the design and implementation of tax.

Keywords: efficiency, value added tax, fiscal policy, JEL Codes: H20, E62. 


\section{INTRODUCCIÓN}

El Impuesto al Valor Agregado (IVA) es muy relevante en cualquier reforma tributaria dada su preponderancia relativa en la estructura impositiva de los países (Gomez Sabaini \& Morán, 2014). En Argentina, y en la mayoría de los países de Latinoamérica, una parte importante de la política recurrentemente propone establecer alícuotas diferenciales a ciertos bienes y servicios que componen la canasta básica de consumo. Algunos estudios de impacto distributivo también han servido para abonar esta teoría, al señalar que el IVA tendría cierta progresividad, cuando el indicador de bienestar es el consumo, como resultado de los efectos positivos de las exenciones y alícuotas reducidas del impuesto al valor agregado en algunos bienes de consumo. En sentido contrario, y con fines recaudatorios, el FMI ha propuesto generalizar el IVA a tasa única eliminando los bienes exentos y a tasa reducida.

Economistas del campo de las finanzas públicas (Bird 2005, Keen, 2013, Mirlless, 2011, Zee, 1995) han abordado cuidadosamente los problemas de diseño e implementación del IVA en los distintos países. En este sentido, han señalado los efectos adversos sobre la eficiencia, la administración tributaria y la evasión que tiene la utilización de tasas diferenciales, bienes exentos y bienes con tasas cero. Sin embargo, en una revisión del diseño del IVA en la mayoría de los países desarrollados y en desarrollo, se observan características similares en cuanto a alejarse de una tasa impositiva única.

Las cuestiones de diseño e implementación en la instrumentación del IVA adquieren importancia dado que es el impuesto al consumo por excelencia a nivel mundial (con excepción de los Estados Unidos). En el año 2016 la recaudación del impuesto en los países de la OECD fue del 6.8\% del PBI y representó el 20.2\% de la recaudación total (en 1975 era del 3.1\% del PBI y el 8.8\% del total). En países de la región, como Chile, el IVA recaudó en 2016 el 8.3\% del PBI y tuvo una participación sobre el total recaudado del $41.2 \%$. En Argentina el impuesto recaudó en el año 2018 un $7.6 \%$ del PBI y representó el $32.6 \%$ del total de recursos tributarios. Como señala Mirlless (2011),

In terms of revenue raised, VAT is by a long distance the most important indirect tax in the UK, and in most other OECD countries. Since its introduction in France in 1954, it has proved an exceptionally successful form of taxation and has been adopted by many countries worldwide, including all OECD countries other than the US. Bird (2010,363) calls it 'unquestionably the most successful fiscal innovation of the last half- 
century... perhaps the most economically efficient way in which countries can raise significant tax revenues.

El objetivo de este trabajo es estudiar los efectos de la existencia de tasas reducidas, bienes a tasa cero y exenciones del IVA en Argentina sobre los precios de los bienes (efectos sobre la eficiencia económica), el impacto distributivo y la eficiencia recaudatoria. Estos temas parecerían estar olvidados, o poco analizados cuando se debate y se realizan estudios sobre la política tributaria del país.

El trabajo se encuentra organizado de la siguiente manera. En la sección I se analiza el diseño del impuesto y como funciona en la práctica la metodología de débitos y créditos. En la sección II se realiza un repaso de la literatura y en la sección III se observa el caso particular de Nueva Zelanda. En la sección IV se presenta el impuesto para Argentina. En la sección V se realiza una estimación de la Eficiencia $\mathrm{C}$ y finalmente se presentan las consideraciones finales.

\section{ACERCA DEL DISEÑO DEL IMPUESTO}

En esta sección se analizan algunas cuestiones relacionadas con el diseño del impuesto. Básicamente, cómo diferentes formas de implementación afectan los precios relativos, la recaudación tributaria y la complejidad de la administración tributaria, según los bienes estén gravados con un IVA a tasa única, tasa cero o exentos.

\section{I.1. Metodología del IVA créditos y débitos}

La recaudación del IVA vía el mecanismo de débitos y créditos implica que el monto a tributar por dicho impuesto sea aquel que surge de la diferencia entre el monto gravado en la venta (débito) respecto del correspondiente a la compra (crédito), destacando que los precios en ambas operaciones discriminan el IVA (es decir, incluyen de manera explícita el impuesto). A efectos de una mejor interpretación del mecanismo descripto, se plantean a modo de ejemplificación numérica seis casos bajo un esquema productivo de un bien en tres etapas, estableciendo a su vez dos supuestos de trabajo: cada etapa de la cadena agrega valor por $\$ 100 \mathrm{y}$ la alícuota general del impuesto es del 10\%. El ejercicio también supone que toda la carga del impuesto recae sobre el consumidor final del bien.

En el caso de la tabla 1, todas las actividades se encuentran gravadas con una alícuota del $10 \%$, por ende la recaudación en cada etapa asciende a $\$ 10$ y acu- 
mula al final de la cadena un monto de $\$ 30$. El monto del impuesto se corresponde con el $10 \%$ del valor agregado en toda la cadena (\$300) y el consumidor final enfrenta un precio de $\$ 330$ (incluido el impuesto).

Tabla 1. Implementación del IVA en tres etapas

Todas las actividades están gravadas a la misma tasa

\begin{tabular}{|c|c|c|c|c|c|}
\hline Concepto & Productor & Mayorista & Minorista & $\begin{array}{l}\text { Recaudación } \\
\text { Total }\end{array}$ & $\begin{array}{c}\text { Precio Final } \\
\text { con IVA }\end{array}$ \\
\hline $\begin{array}{l}\text { (1) Ventas } \\
\text { (excluyendo } \\
\text { IVA) }\end{array}$ & 100 & 200 & 300 & & \\
\hline $\begin{array}{l}\text { (2) Compras } \\
\text { (excluyendo } \\
\text { IVA) }\end{array}$ & 0 & 100 & 200 & & \\
\hline $\begin{array}{l}\text { (3) IVA } \\
\text { sobre ventas } \\
{[10 \% \text { de (1)] }}\end{array}$ & 10 & 20 & 30 & & \\
\hline $\begin{array}{l}\text { (4) Crédito } \\
\text { por compras } \\
{[10 \% \text { de }(2)]}\end{array}$ & 0 & 10 & 20 & & \\
\hline $\begin{array}{l}\text { (5) IVA neto } \\
\text { [(3)-(4)] }\end{array}$ & 10 & 10 & 10 & 30 & 330 \\
\hline
\end{tabular}

Fuente: elaboración propia.

La introducción de alícuotas al 0\% y/o exenciones impositivas presentan modificaciones al caso analizado precedentemente. En la tabla 2 se plantea un escenario en donde el comercio minorista está gravado a tasa cero. Puede observarse que la situación del productor y el mayorista no dista respecto de aquella presentada en la tabla 1 (alícuota uniforme), pero en la etapa final se produce una devolución completa del tributo $(\$ 20)$. Dicha situación es la resultante de la operación conjunta del sistema de créditos y débitos fiscales con una tasa cero en la etapa final. De esta manera, el minorista tiene la posibilidad de reclamar saldos a favor dado que sus ventas están sujetas a tasa cero y conserva los créditos por compras, erosionando completamente la recaudación final del impuesto. En este caso, el consumidor final enfrenta un precio de $\$ 300$, el cual se condice con el valor agregado total. El ejemplo típico son las exportaciones que están gravadas a tasa cero. La agencia 
recaudadora le devuelve el impuesto al exportador al momento de realizar la venta al exterior. Ello implica un gran esfuerzo de control por parte de la autoridad tributaria y se expone a los riesgos de fraude que esta operación conlleva.

Tabla 2. Implementación del IVA en tres etapas

El comercio minorista está gravado a tasa cero

\begin{tabular}{|c|c|c|c|c|c|}
\hline Concepto & Productor & Mayorista & Minorista & $\begin{array}{l}\text { Recaudación } \\
\text { total }\end{array}$ & $\begin{array}{l}\text { Precio final } \\
\text { con IVA }\end{array}$ \\
\hline $\begin{array}{l}\text { (1) Ventas } \\
\text { (excluyendo } \\
\text { IVA) }\end{array}$ & 100 & 200 & 300 & & \\
\hline $\begin{array}{l}\text { (2) Compras } \\
\text { (excluyendo } \\
\text { IVA) }\end{array}$ & 0 & 100 & 200 & & \\
\hline $\begin{array}{l}\text { (3) IVA } \\
\text { sobre ventas } \\
{[10 \% \text { de (1)] }}\end{array}$ & 10 & 20 & 0 & & \\
\hline $\begin{array}{l}\text { (4) Crédito } \\
\text { por compras } \\
{[10 \% \text { de }(2)]}\end{array}$ & 0 & 10 & 20 & & \\
\hline $\begin{array}{l}\text { (5) IVA neto } \\
{[(3)-(4)]}\end{array}$ & 10 & 10 & -20 & 0 & 300 \\
\hline
\end{tabular}

Fuente: elaboración propia.

Un punto de análisis resulta de comparar los primeros dos casos expuestos. En dicha comparación se observa que frente a un cambio de política tributaria de un escenario de alícuota uniforme a otro con alícuota cero en la etapa final permitiría obtener una reducción del precio equivalente a la alícuota del impuesto (10\%). Es decir el precio final es de $\$ 300$.

En la tabla 3 se analiza el caso de exenciones en la etapa minorista. Es decir el contribuyente está exento del impuesto solo en la etapa final. La recaudación del impuesto se realiza en las etapas anteriores, los créditos y débitos fiscales de dichas etapas se trasladan al precio final del producto. Por lo tanto, la recaudación efectiva de $\$ 20$ se corresponde con los montos recaudados sobre el valor agregado en las primeras etapas. El precio final al que se vende el bien es de $\$ 320$ (se modifican precios relativos). Como puede observarse la reducción del precio final no es del 10\% como algunos equivocadamente suponen, la reducción asciende a solo el 3\% (320 sobre 330). 
Además se complejiza la administración tributaria al perderse el control cruzado de débitos y créditos entre los contribuyentes y aumenta los riesgos de evasión impositiva.

Tabla 3. Implementación del IVA en tres etapas

El comercio minorista está exento

\begin{tabular}{|c|c|c|c|c|c|}
\hline Concepto & Productor & Mayorista & Minorista & $\begin{array}{c}\text { Recaudación } \\
\text { total }\end{array}$ & $\begin{array}{c}\text { Precio final } \\
\text { con IVA }\end{array}$ \\
\hline $\begin{array}{l}\text { (1) Ventas } \\
\text { (excluyendo } \\
\text { IVA) }\end{array}$ & 100 & 200 & 300 & & \\
\hline $\begin{array}{l}\text { (2) Compras } \\
\text { (excluyendo } \\
\text { IVA) }\end{array}$ & 0 & 100 & 200 & & \\
\hline $\begin{array}{l}\text { (3) IVA } \\
\text { sobre ventas } \\
{[10 \% \text { de }(1)]}\end{array}$ & 10 & 20 & & & \\
\hline $\begin{array}{l}\text { (4) Crédito } \\
\text { por compras } \\
{[10 \% \text { de }(2)]}\end{array}$ & 0 & 10 & & & \\
\hline $\begin{array}{l}\text { (5) IVA neto } \\
{[(3)-(4)]}\end{array}$ & 10 & 10 & & 20 & 320 \\
\hline
\end{tabular}

Fuente: elaboración propia.

En la tabla 4 se aplica la exención al productor mayorista. Esto genera un "efecto cascada" del impuesto al interrumpir el sistema de débitos y créditos a mitad de la cadena, produciéndose un traslado a precios sobre el cual posteriormente se aplicará la alícuota correspondiente. El resultado del caso bajo estudio arroja la mayor recaudación (\$41) y precio final (\$341) en términos comparados, por lo que es de esperar que la distorsión del impuesto sea mayor debido al diseño planteado. La realización de un análisis pormenorizado del caso posibilita apreciar el incremento en el precio de venta en la etapa intermedia respecto al caso precedentemente analizado (\$210 frente a \$200). La diferencia de \$10 es el resultado de cargar sobre el precio de venta el monto del impuesto que queda exento del sistema de débitos y créditos por parte del mayorista. Posteriormente, dado que el mayorista no discrimina el impuesto al realizar la venta al minorista este último no contará con un crédito fiscal para descontar de los débitos en que incurra. En el ejemplo el monto del IVA sobre ventas asciende a \$31 el cual surge de aplicar la alícuota del 
$10 \%$ al valor de las ventas en la etapa final (\$310). El proceder antes descripto totaliza con una recaudación de $\$ 41$ en concepto de IVA. La interrupción de la cadena de créditos fiscales tuvo como implicancia establecer "impuestos sobre impuestos" sobre el valor agregado del productor (permite recaudar $\$ 11$ adicionales).

Tabla 4. Implementación del IVA en tres etapas

El comercio mayorista está exento

\begin{tabular}{|c|c|c|c|c|c|}
\hline Concepto & Productor & Mayorista & Minorista & $\begin{array}{c}\text { Recaudación } \\
\text { total }\end{array}$ & $\begin{array}{c}\text { Precio final } \\
\text { con IVA }\end{array}$ \\
\hline $\begin{array}{l}\text { (1) Ventas } \\
\text { (excluyendo } \\
\text { IVA) }\end{array}$ & 100 & 210 & 310 & & \\
\hline $\begin{array}{l}\text { (2) Compras } \\
\text { (excluyendo } \\
\text { IVA) }\end{array}$ & 0 & 100 & 210 & & \\
\hline $\begin{array}{l}\text { (3) IVA } \\
\text { sobre ventas } \\
{[10 \% \text { de }(1)]}\end{array}$ & 10 & & 31 & & \\
\hline $\begin{array}{l}\text { (4) Crédito } \\
\text { por compras } \\
{[10 \% \text { de }(2)]}\end{array}$ & 0 & & & & \\
\hline $\begin{array}{l}\text { (5) IVA neto } \\
{[(3)-(4)]}\end{array}$ & 10 & & 31 & 41 & 341 \\
\hline
\end{tabular}

Fuente: elaboración propia.

En todos los casos expuestos, el análisis ha sido focalizado hacia aspectos cuantitativos, pero es menester destacar aspectos cualitativos que presentarían importantes consecuencias en el diseño de la política tributaria como en la administración del tributo. Un ejemplo de ello sería el establecimiento de una alícuota uniforme para el IVA, medida que simplificaría la fiscalización del impuesto dado que se reducirían los incentivos hacia comportamientos oportunistas (incentivos al fraude fiscal). Existe una clara relación entre tratamientos preferenciales y evasión fiscal (Slemrod, 1988).

Por otra parte, se observan importantes cambios en los precios relativos de los bienes según los distintos diseños del impuesto que se adopten con significativos efectos sobre la eficiencia económica. 
Una alternativa de análisis se presenta en el caso de alícuotas diferenciales en las distintas etapas de la cadena. En la tabla 5 se presenta el caso en que la alícuota es del $5 \%$ en la etapa mayorista y minorista (10\% en la producción). La recaudación es igual al 5\% del valor agregado total, es decir, \$15. La alícuota de la etapa minorista define el monto recaudado. El precio final se ve afectado proporcionalmente al cambio de la tasa del IVA.

Tabla 5. Implementación del IVA en tres etapas

Alícuotas diferenciales: $5 \%$ en las etapas mayorista, minorista y $10 \%$ producción

\begin{tabular}{|c|c|c|c|c|c|}
\hline Concepto & Productor & Mayorista & Minorista & IVA total & $\begin{array}{c}\text { Precio final } \\
\text { con IVA }\end{array}$ \\
\hline $\begin{array}{l}\text { (1) Ventas } \\
\text { (excluyendo } \\
\text { IVA) }\end{array}$ & 100 & 200 & 300 & & \\
\hline $\begin{array}{l}\text { (2) Compras } \\
\text { (excluyendo } \\
\text { IVA) }\end{array}$ & 0 & 100 & 200 & & \\
\hline $\begin{array}{l}\text { (3) IVA } \\
\text { sobre ventas } \\
{[\% \text { de (1)] }}\end{array}$ & 10 & 10 & 15 & & \\
\hline $\begin{array}{l}\text { (4) Crédito } \\
\text { por compras } \\
{[\% \text { de }(2)]}\end{array}$ & 0 & 10 & 10 & & \\
\hline $\begin{array}{l}\text { (5) IVA neto } \\
{[(3)-(4)]}\end{array}$ & 10 & 0 & 5 & 15 & 315 \\
\hline
\end{tabular}

Fuente: elaboración propia.

Por último en la tabla 6 se presenta el caso de una alícuota más alta en la etapa mayorista en relación con las establecidas en la etapa productiva y minorista. En este ejemplo, la recaudación sigue siendo igual a la alícuota del comercio minorista por el valor agregado $(\$ 15)$. Pero esto solo ocurre si al comercio minorista se le devuelven $\$ 5$ de crédito fiscal. Como generalmente esto no ocurre debido a que es muy difícil identificar ese crédito para las administraciones tributarias, el minorista asume ese crédito como un costo, la recaudación total es de $\$ 20$ y el precio final asciende a $\$ 320$. Como se verá más adelante, este ejemplo es muy relevante para el caso argentino en donde los servicios de gas, luz y agua tienen una alícuota agravada del $27 \%$ para todas las ventas que no son domiciliarias. 
Tabla 6. Implementación del IVA en tres etapas

Alícuotas diferenciales: $5 \%$ en la producción, $10 \%$ mayorista y 5\% minorista

\begin{tabular}{|c|c|c|c|c|c|}
\hline Concepto & Productor & Mayorista & Minorista & IVA total & $\begin{array}{c}\text { Precio final } \\
\text { con IVA }\end{array}$ \\
\hline $\begin{array}{l}\text { (1) Ventas } \\
\text { (excluyendo } \\
\text { IVA) }\end{array}$ & 100 & 200 & 300 & & \\
\hline $\begin{array}{l}\text { (2) Compras } \\
\text { (excluyendo } \\
\text { IVA) }\end{array}$ & 0 & 100 & 200 & & \\
\hline $\begin{array}{l}\text { (3) IVA } \\
\text { sobre ventas } \\
{[\% \text { de }(1)]}\end{array}$ & 5 & 20 & 15 & & \\
\hline $\begin{array}{l}\text { (4) Crédito } \\
\text { por compras } \\
{[\% \text { de }(2)]}\end{array}$ & 0 & 5 & 20 & & \\
\hline $\begin{array}{l}\text { (5) IVA neto } \\
{[(3)-(4)]}\end{array}$ & 5 & 15 & -5 & 15 & 315 \\
\hline
\end{tabular}

Fuente: elaboración propia.

\section{UN REPASO POR LA LITERATURA}

El análisis de los tratamientos especiales en el IVA ha producido una amplia y relevante literatura. A pesar del consenso académico general acerca de la conveniencia de establecer tasas del impuesto uniformes a lo largo de la cadena de valor y una base imponible amplia, es una práctica común en los gobiernos del mundo no seguir esta regla (Ebrill et al., 2002; de la Feria y Krever, 2013; OECD, 2018; Annacondia, 2019; Ernst y Young, 2019). Estos alejamientos del diseño ideal traen problemas de eficiencia económica, recaudación tributaria y no parecen ser el mejor instrumento para mejorar la distribución del ingreso ${ }^{1}$.

1 Desde la teoría de la imposición óptima (regla de Ramsey-Feldstein) habría que imponer a los bienes con demandas más inelásticas, complementarios del ocio y que consumen relativamente más los más ricos. Sin embargo, desde la política económica se reconocen argumentos que conducen a apartarse de esta regla como los problemas de administración tributaria, neutralidad impositiva, simplicidad, lobby, entre otros. 


\section{II.1. Efectos sobre la eficiencia económica}

Uno de los principales inconvenientes que pueden generarse producto del apartamiento de la regla de tratamiento uniforme en el IVA está relacionado con la eficiencia económica y las pérdidas de bienestar. La existencia de exenciones o tasas diferenciales en algún eslabón de la cadena de valor incentiva a las firmas a autoabastecerse (Bird et al., 2005; Mirrless, 2011; Harris et al., 2018) con el fin de proveerse de insumos con menor carga impositiva. Adicionalmente, estos mecanismos pueden crear poder de mercado en favor de firmas con menor carga de IVA que otras, profundizando los costos en términos de eficiencia económica. A su vez, Agha y Haughton (1996) establecen que la distorsión en los precios relativos de los bienes producto de la inexistencia de un IVA con tratamiento uniforme puede exacerbarse si el Estado no está dispuesto a reasignar recursos. Es decir, de acuerdo a los autores, será necesario incrementar las alícuotas con las que se gravan otros bienes si el Estado pretende aplicar tratamientos especiales sin descuidar su recaudación. Por otro lado, Ebrill et al. (2001) resalta las distorsiones generadas por los tratamientos no uniformes en lo que respecta a la provisión de insumos, generando incentivos al autoabastecimiento. En el mismo sentido, los tratamientos especiales podrían propiciar la elección de proveedores menos eficientes pero cuya producción soporta menores cargas de IVA.

Otra serie de problemas de eficiencia económica asociados a la inexistencia de tratamientos uniformes del IVA se encuentran en Bird et al. (2005) y Mirrless (2011). El primero de ellos está relacionado con la existencia de un exemption creep (Gauthier \& Gersovitz, 1997). Este término hace referencia al hecho de que, una vez que cierto eslabón de la cadena de valores recibe exenciones o rebajas de alícuotas, se vuelve muy complejo quitarle esas ventajas en el futuro, generándose cierto grado de dependencia de las firmas a ellas e, incluso, mecanismos de lobby (Abramovsky et al., 2017). Un inconveniente adicional, en términos de eficiencia económica, se encuentra asociado a los tratamientos especiales del IVA en los bienes provistos públicamente. En algunos casos la frontera que divide los bienes públicos y privados es un tanto difusa por lo que se dificulta establecer qué tipo de tratamiento de IVA asignar a cada bien. Por otro lado, el tratamiento especial también sesga los incentivos dentro del ámbito público, generando estímulos a la producción propia en detrimento de la producción privada, lo cual no necesariamente es la opción más eficiente en términos económicos. Es por estas razones, que la literatura (Aujean et al., 1999; Gendron, 2005; Bird y Gendron, 2007; Copenhagen Economics and KPMG, 2011) recomienda que la base imponible del IVA uniforme también abarque bienes provistos de manera pública. 


\section{II.2. Efectos sobre la recaudación y evasión}

Los problemas en términos de costos administrativos y recaudatorios de los tratamientos especiales del IVA son también analizados ampliamente por la literatura. En términos generales, existe consenso académico en proponer una base del impuesto amplia con tasa uniforme, lo que facilita y hace transparente la administración del impuesto (Cnossen, 2004; Bird et al., 2005; Keen y Smith, 2006). A su vez, dicha recomendación parece resultar aún más relevante para países en desarrollo cuyos sistemas tributarios son menos eficientes y poseen bases imponibles relativamente menores a causa de contar con tasas de informalidad más altas (Ahmad \& Best, 2012; Anton, et al., 2013; Abramovsky et al., 2017). En general, los costos en términos de recaudación por apartarse de estas recomendaciones suelen ser altos. La práctica usual en la literatura para medir estos costos radica en estimar la relación entre la recaudación real por IVA y la recaudación teórica derivada de aplicar la tasa del impuesto al consumo agregado de la economía (eficiencia C). Para países emergentes, el Fondo Monetario Internacional estima esa tasa en alrededor de $50 \%$, es decir, solo se alcanza la mitad de la recaudación potencial del IVA (IMF, 2010).

Si bien las pérdidas recaudatorias del IVA no pueden ser atribuibles enteramente a problemas de evasión y dificultades administrativas producto de tratamientos especiales en el tributo, estos inconvenientes parecen representar una gran proporción de las ineficiencias recaudatorias (James, 2015). Existen una serie de trabajos tendientes a estimar la proporción de la recaudación tributaria de IVA que se pierde a causa de tratamientos especiales. Algunos de ellos concentran su análisis en el sector financiero. En este sentido, Huizinga (2002) encuentra que la supresión de los tratamientos no uniformes en ese sector implicarían un aumento en la recaudación de IVA financiero de $4.7 \%$ en la Unión Europea. En estudios más recientes, de la Feria (2010) establece que el incremento en la recaudación tributaria del IVA podría ser de hasta $15 \%$, respecto a la recaudación actual del tributo en dicho sector, si se eliminaran las exenciones existentes en el sector financiero; mientras que Adam et al. (2011) estiman dicho número en alrededor de $2.13 \%$ en España, 2.7\% en Italia, 3.46\%, en Alemania y $4.32 \%$ en Francia. En sentido contrario, Buettner y Erbe (2014) analizan el caso alemán y estiman una pérdida de recaudación por IVA financiero de alrededor $1.3 \%$.

Agha y Haughton (1996) utilizan datos para 17 países de la Unión Europea encontrando evidencia empírica que sugiere que la existencia de tratamientos no uniformes en el IVA está asociada negativamente con la recaudación tributaria. A su vez, Bogetic \& Hassan (1993) y Jack (1996) utilizan datos de Europa Central y del Este encontrando que cuanto menos uniforme es el IVA de los países, mayor es 
la pérdida en términos de recaudación fiscal. Más recientemente, Reckon (2009) realiza una comparación entre recaudación real y potencial en la Unión Europea entre 2000 y 2006 para determinar la proporción de la diferencia entre ambos conceptos que es atribuible a fraude y evasión. El trabajo encuentra que la recaudación por IVA podría incrementarse entre $12 \%$ y $14 \%$ si se evitaran estos casos. A su vez, Barbone et al. (2012) establece que el alejamiento del tratamiento uniforme del IVA representa la principal razón que fomenta situaciones de fraude y evasión. Dicho trabajo también ofrece una revisión de literatura, encontrando que la pérdida de recursos del Estado asociada a tratamientos especiales del IVA en la Unión Europea es de un 14\% en promedio. En el mismo sentido, Keen (2013) realiza una descomposición de la brecha entre la recaudación por IVA efectiva y potencial, asociada a una situación de tratamiento uniforme e inexistencia de evasión fiscal. Analizando países de la Unión Europea, el autor encuentra evidencia que indica que las pérdidas de recaudación producto de la aplicación de tasas no uniformes y exenciones son notoriamente más grandes que aquellas vinculadas puramente a evasión fiscal.

En definitiva, la literatura identifica la existencia de una relación entre tratamientos diferenciales y evasión, lo cual repercute en los ingresos del fisco. Tal como se señala en Mirrless (2011), el alejamiento del tratamiento uniforme puede generar incentivos a la evasión fiscal en detrimento de la recaudación impositiva. En particular, las firmas pueden sacar ventaja de la dificultad existente en distinguir entre actividades sujetas o no a tratamientos especiales tanto declarando dedicarse a actividades exentas o con alícuotas menores, como proveyéndose de insumos con o sin tratamiento especiales.

\section{II.3. Efectos sobre aspectos distributivos}

Otro de los argumentos para validar los tratamientos especiales en el IVA se basa en cuestiones distributivas. Al ser el IVA un impuesto que generalmente se supone que recae sobre el consumidor final, se esgrime que la existencia de exenciones o tasas diferenciales sobre algunos bienes podría ayudar a hacer la distribución del ingreso más igualitaria (Bird et al., 2005; Harris et al., 2018). Sin embargo, la literatura advierte sobre algunos inconvenientes para considerar al IVA como mecanismo redistributivo. James (2015) menciona que el IVA no fue diseñado para tales fines y que la mejor opción, para minimizar distorsiones, radica en complementar este impuesto con transferencias a hogares con menores ingresos (Mirrless, 2011; Ahmad \& Best, 2012; Anton, et al., 2013; Harris et al., 2018). A su vez, Cnossen (2004) asevera que la existencia de tratamientos diferenciales en el IVA con fines redistributivos no alcanza a compensar los costos en términos de 
eficiencia y recaudación que implican. Adicionalmente, Ebrill et al. (2001) y Abramovsky et al. (2017) explican que el argumento de establecer tasas diferenciales sobre bienes de primera necesidad no es suficiente, dado que usualmente existen fallas de focalización asociadas a ese tratamiento del IVA. En tanto, Harris et al. (2018) argumenta que estos problemas pueden agudizarse en países en vías de desarrollo, dada la menor calidad administrativa de sus sistemas tributarios.

La evidencia empírica existente acerca de la regresividad del IVA en Argentina no resulta concluyente. Usualmente los trabajos encuentran que dicho tributo es regresivo medido por ingreso, pero neutro al medirlo por consumo. Para el caso argentino, se arriba a esta conclusión en los trabajos de Gasparini (1998), Artana et al. (2015) y Fernández Felices et al. (2016). Es decir, el aspecto regresivo del IVA depende crucialmente del indicador de bienestar. No obstante, cabe aclarar que la metodología utilizada en estos estudios para el análisis de los efectos del IVA en la distribución del ingreso se concentra en estudiar solo la etapa final de la cadena de valor. La práctica usual radica en asignar la carga del impuesto a lo largo de la distribución del ingreso, de acuerdo con la proporción del consumo de cada quintil según surge de la Encuesta Nacional de Gastos de Hogares. El análisis requeriría una complejidad mayor. Como se mostró en la sección I, un bien exento beneficia solo al último eslabón de la cadena productiva, quedando gravada todas las etapas anteriores. Es decir, la exención no implica que la rebaja de la carga impositiva sea igual al precio final menos la alícuota impositiva. Un análisis completo en este sentido debería incluir a toda la cadena de valor a partir de datos de valor agregado de cada etapa (Fiel 1998, Mirrless 2011).

En definitiva, existe consenso en la literatura acerca de que resulta más apropiado un IVA con base imponible amplia, sin exenciones y con tratamientos uniformes para todos los bienes, incluso aquellos con características de bienes públicos. Las razones para estas recomendaciones están ligadas al hecho de que, de esta forma, se minimizan pérdidas de eficiencia, se simplifica la administración del tributo y se incrementa la recaudación. A su vez, la literatura concuerda en que potenciales inconvenientes distributivos asociados al IVA deberían compensarse con esquemas de transferencia y no introduciendo tratamientos especiales en el impuesto.

\section{EL CASO DE NUEVA ZELANDA}

La legislación tributaria del IVA en Nueva Zelanda es un caso emblemático, ampliamente citado en la literatura, en donde se prioriza la minimización de los tratamientos diferenciales del impuesto. Reconocido como el país que mejor 
administra el IVA, en su sistema tributario prácticamente no existen exenciones a dicho impuesto (Dickson \& White, 2011). De acuerdo a James (2015), las únicas excepciones a una base amplia se encuentran en casos muy puntuales tales como servicios financieros a consumidores, algunos servicios residenciales y bienes y servicios provistos por organizaciones sin fines de lucro. Dicho país carga con IVA uniforme a productos y servicios que en otros sistemas tributarios, incluso pertenecientes a la OECD, comúnmente reciben tratamientos diferenciales tales como salud, educación, alimentación, ropa y libros (Poddar, 2005; Bird et al., 2005; James, 2015). Adicionalmente, Nueva Zelanda es uno de los pocos países del mundo, junto con Australia, que evitan distorsionar el tratamiento uniforme del IVA, incluso sobre bienes públicos (Mirrless, 2011). De esta manera, el impuesto al valor agregado en Nueva Zelanda sigue el enfoque convencional por lo que los costos del impuesto en términos de eficiencia resultan mínimos, generando escasas distorsiones a lo largo de la cadena de valor. No obstante, se tienen en cuenta posibles efectos distributivos adversos que podría generar el IVA. Nueva Zelanda, un país con niveles de desigualdad bajos, representa un claro ejemplo acerca de la posibilidad de enfrentar posibles inconvenientes distributivos producto de impuestos al valor agregado de una forma focalizada y poco distorsiva. De acuerdo con James (2015), los posibles problemas distributivos asociados a poseer un IVA de base amplia y tratamiento uniforme son mitigados en Nueva Zelanda (y también en Canadá) mediante transferencias hacia contribuyentes de bajos ingresos. Si bien este mecanismo implica la posibilidad de poder identificar a dicho grupo poblacional, posee la ventaja de estar mejor focalizado que los tratamientos diferenciales de IVA. Adicionalmente, estas transferencias se encuentran diseñadas de manera independiente del impuesto en sí, por lo que no generan distorsiones ni atentan contra los objetivos generales del IVA.

Como resultado de la existencia de un IVA que prácticamente no se aparta de las recomendaciones de la literatura, Nueva Zelanda es identificable como el país con mejor desempeño en el impuesto al valor agregado en el mundo. No solo se evitan distorsiones y pérdidas de eficiencia económica, sino que también la recaudación fiscal por IVA en dicho país, en términos de PBI, es una de las más altas del mundo (James, 2015; Artana et al., 2015; OECD, 2018). A su vez, de acuerdo a OECD (2018), Nueva Zelanda posee una relación entre recaudación potencial y efectiva de IVA muy cercana a 1 y así ha sido desde la implantación de dicho tributo. 


\section{IMPLEMENTACIÓN DEL IVA EN ARGENTINA}

\section{IV.1. La ley vigente y sus efectos}

En Argentina el IVA es un tributo tipificado como "IVA consumo" en donde las inversiones están desgravadas. El impuesto grava todas las ventas, sean estas mayoristas o minoristas, deduciendo el monto del impuesto pagado en etapas intermedias, en un sistema de créditos y débitos fiscales que permite el control cruzado entre los contribuyentes. Además el IVA grava los bienes y servicios en "destino", desgrava las exportaciones y grava las importaciones. Dado que el valor final del bien es igual a la suma del valor agregado en cada etapa, la base imponible es similar a la de un impuesto a las ventas finales, pero recaudado en cada etapa de producción y comercialización.

De acuerdo al texto ordenado de la Ley n. ${ }^{\circ} 23349$ de Impuesto al Valor Agregado, se establece que el IVA tiene operaciones exentas del gravamen y, a su vez, contempla tratamientos alicuotarios diferenciales para un conjunto de bienes y servicios. Como fuera señalado, este diseño no es ajeno al que utilizan los países de la OECD con un conjunto de tratamiento especiales por razones sociales (salud, educación, caridad), prácticas (servicios financieros y seguros) e históricas (OECD 2018).

El fundamento de los tratamientos diferenciales en la legislación argentina podrían fundarse en: i) razones distributivas (por ejemplo en el caso del alimentos, transporte, vivienda), ii) provisión de bienes meritorios (salud, educación, actividades culturales) y iii) dificultades para cobrar el IVA a ciertos sectores (seguros, pequeños contribuyentes) $)^{2}$.La tasa general del IVA es del $21 \%$, existiendo una tasa más alta (del 27\%) para las ventas de gas, energía eléctrica y aguas reguladas por medidor y telecomunicaciones. A su vez tienen una tasa reducida del $50 \%$ de la alícuota $(10.5 \%)$ las ventas, locaciones o importaciones relacionadas con las actividades agrícolas ganaderas, los productos elaborados en base a harina de trigo antes de su comercialización y el tratamiento de los residuos sólidos.

Esta estructura de exenciones y alícuotas agravadas (27\%) y reducidas (10.5) alejan el diseño del impuesto de un esquema neutral entre sectores, que

2 Un argumento para la existencia de tratamientos diferenciales es la existencia de externalidades. Alícuotas más altas podrían justificarse en bienes con externalidades negativas (por ejemplo, cigarrillos) o más bajas en aquellos con externalidades positivas (por ejemplo, investigación y desarrollo). En general, la literatura ha reconocido a los impuestos específicos como más eficientes para para corregir estas distorsiones. 
evite las distorsiones de precios relativos, aumente el gasto tributario ${ }^{3}$ y complique la actuación y el control de la agencia recaudadora.Siguiendo la sección I, si el objetivo es mejorar la distribución del ingreso, los efectos de las exenciones en la etapa final del bien o servicio tienen un efecto limitado sobre los precios finales de los productos. Este tipo de tratamiento impositivo beneficia también a los quintiles más altos de la distribución del ingreso. La literatura ha sido muy contundente en el sentido que es mejor transferir ingresos a la población más vulnerable en lugar de exenciones tributarias que limitan la base imponible y cuyos beneficios se filtran sobre el resto de los quintiles de la distribución del ingreso. En esta misma línea CEPAL (2019) señala que

[los] gastos tributarios buscan aminorar los efectos negativos de un impuesto sobre un grupo de contribuyentes de menores recursos, aunque no siempre dicha medida sea la forma más eficiente de cumplir ese objetivo. Esto se ve frecuentemente en el caso del impuesto sobre el valor agregado, en que se aplica una exención o una tasa cero a los productos de la canasta básica con el fin de apoyar a los hogares con menores ingresos.

Algo similar ocurre con las tasas reducidas (por ejemplo 10.5\%). Aquí la diferencia central es que una tasa reducida en la etapa final de la cadena es la que determina el nivel de imposición real del bien (sección I). Es decir, si en el proceso productivo el bien tuvo alícuotas mayores, todos los créditos acumulados en las etapas previas se cancelan en la fase final. La alícuota efectiva es la que se fija al final de la cadena. Este no sería el caso si los créditos acumulados no pueden descargarse en la venta final del producto. El impacto distributivo es incierto.

Los bienes gravados a tasa cero permiten que se reintegren todos los créditos de las etapas previas. Es el caso típico de las exportaciones. El gran problema es de administración tributaria. El riesgo de fraude es muy alto (existe la posibilidad de compraventa de facturas apócrifas para obtener crédito fiscal). En el mes de julio de 2019 se le otorgó un tratamiento de este tipo a la industria editorial y gráfica (imprentas, editoriales y distribuidoras). El punto es como diferenciar el papel que se destina a la industria gráfica de aquel que se utiliza para envoltorios de productos en general o cualquier otro destino. Nuevamente los riesgos de fraude son altos.

3 El gasto tributario es una transferencia de recursos públicos realizada mediante reducciones de las obligaciones impositivas en relación con un marco de referencia, en vez de ser efectuada a través de un gasto público directo (OECD, 2018). 
También existe un tratamiento especial para los pequeños contribuyentes que están exentos del IVA. Las razones están fundadas en los costos de cumplimiento y de fiscalización por parte de la agencia recaudadora. El problema en Argentina es que el límite superior (threshold) para poder actuar como pequeño contribuyente (monotributista) parece muy elevado y conduce a comportamientos estratégicos para no superar esos límites (enanismo fiscal) ${ }^{4}$. Sin embargo, el avance en la mayoría de los países hacia la facturación electrónica, podría acotar este problema. La formalización del comercio reduciría así el espacio para la evasión y elusión fiscal del IVA. Al mismo tiempo, el cruce de información proveniente de la facturación electrónica, registros administrativos y otras bases de datos permitiría la detección de irregularidades en las declaraciones juradas de los contribuyentes, aun de los más pequeños.

La alícuota agravada del $27 \%$ en los servicios de luz, agua y gas para consumos no residenciales no pareciera tener una explicación clara. Encarece relativamente insumos básicos y no tiene efectos recaudatorios importantes ya que los créditos fiscales pueden descontarse en la etapa siguiente. La excepción es el caso de los monotributistas (comercio y servicios). En este caso pagan estos servicios con tasas de IVA más elevadas y no tienen forma de descargar los créditos fiscales, de manera que pasa a constituir un costo más elevado.

En la reforma tributaria de 2017 se incluyeron en la base imponible del IVA a los servicios digitales prestados por sujetos residentes o domiciliados en el exterior en la medida que su utilización o explotación efectiva se lleve a cabo en el país. Este es un paso importante para ampliar la base tributaria dado el sostenido incremento de este tipo de operaciones comerciales en la que existía cierto grado de incertidumbre acerca de su tratamiento.

\section{EFICIENCIA “C”, GASTO TRIBUTARIO Y EVASIÓN}

Una forma de analizar el comportamiento del IVA es mediante el concepto de Eficiencia "C" (EC), que mide la relación entre lo efectivamente recaudado y lo que se debería haber recaudado dada la tasa impositiva general del tributo y la base imponible.

4 En la literatura también conocido como Notch (Schwab y Oates, 2015).

5 Un monotributista paga entre un $4 \%$ y $11 \%$ de sus ingresos. En tanto, un responsable inscripto tributa entre un $25 \%$ y $45 \%$. https://www.lanacion.com.ar/economia/lo-mejor-y-lo-peor-de-las-politicastributarias-de-los-candidatos-presidenciales-nid2269489. 
Cabe destacar que este indicador no pretende evaluar el problema en términos de bienestar, ya que un coeficiente de EC del 100\% no necesariamente indica un IVA con mayor eficiencia (este resultado podría ser por ejemplo consecuencia de la no devolución de los créditos fiscales de IVA a los exportadores). Sin embargo, es una aproximación para evaluar la neutralidad del IVA como un instrumento de imposición sobre el consumo doméstico. Un impuesto a tasa única sin exenciones y tratamiento especiales mejoraría el Coeficiente $\mathrm{C}$, a la vez que reduciría la pérdida de bienestar. La referencia de un IVA a tasa uniforme es una forma muy simple y práctica como benchmark para analizar el comportamiento del tributo.

El desempeño de este indicador en cada país puede ser explicado por razones estructurales como el grado de urbanización, apertura económica, participación de la agricultura en el producto, así como por la tasa impositiva, los costos de cumplimiento, la capacidad de auditoría de la agencia recaudatoria, las fluctuaciones en el patrón de consumo, el ciclo económico, entre otras (Junji Ueda, 2017).

En términos de la construcción del indicador, el denominador es clave. Un IVA consumo donde los bienes y servicios se gravan en destino, el denominador es igual al consumo de los residentes: se adicionan las importaciones y se excluyen las exportaciones.

La información del consumo doméstico (neto de impuestos) se obtiene de las cuentas nacionales. El problema que surge para la comparación internacional es que la construcción de las mismas no siempre es enteramente homogénea.

En el caso de Argentina, como puede verse en la tabla 7, de las cuentas nacionales se obtiene la base imponible del impuesto. A partir de esta base y la tasa impositiva general del IVA (21\%) puede construirse el denominador del coeficiente de EC. A su vez, a partir de información de la agencia recaudadora nacional (AFIP) se dispone de las declaraciones juradas de los contribuyentes con los créditos y débitos por sectores de actividad y el impuesto finalmente pagado. El resultado se muestra en la última fila de la tabla. La eficiencia C en el 2017 asciende a 47\%67.

6 Un análisis de este indicador para el período 2004-2017 muestra que las variaciones en el tiempo son poco significativas. La Eficiencia $\mathrm{C}$ es relativamente estable con un leve crecimiento (entre el 43 y $47 \%$ ) en un período en donde se observan etapas bien diferentes en el ciclo económico (expansión del 2004-2010 y estancamiento 2011-2017).

7 Artana (2015) estima la eficiencia C para Argentina en 45.3\% (año 2012). 
Tabla 7. Estimación de la Eficiencia "C" Argentina (Año 2017)

\begin{tabular}{lc}
\hline Concepto & Montos (en millones de pesos) \\
\hline $\begin{array}{l}\text { Consumo más importaciones menos } \\
\text { exportaciones (sin impuestos) }\end{array}$ & 7835395 \\
\hdashline $\begin{array}{l}\text { IVA teórico (21\%) } \\
\text { Impuesto al valor agregado recaudado (neto de } \\
\text { devoluciones) }\end{array}$ & 7645433 \\
\hline Eficiencia "C" & $47 \%$ \\
\hline
\end{tabular}

Fuente: elaboración propia sobre la base de AFIP e INDEC.

La divergencia de la Eficiencia C estimada con una del 100\% está explicada básicamente por dos razones: i) existen bienes o servicios exentos o a tasas reducidas, dificultades para cobrar el impuesto a los pequeños contribuyentes o problemas en la implementación del tributo, ii) evasión de los contribuyentes.

En los países de la OECD las estimaciones de la Eficiencia $\mathrm{C}$ es superior al caso Argentino: $56 \%$ en promedio (tabla 8 ), lo que revela que hay un amplio margen para mejorar el desempeño del impuesto.

En estos países, la divergencia con la recaudación teórica está explicada, en primer lugar, por el gasto tributario y, en menor medida, la evasión, según se muestra en la tabla. Pero ambos conceptos (evasión y gasto tributario) están estrechamente relacionados. Como señala Slemrod (1988), los gastos tributarios generan espacio para la evasión y la elusión fiscal. La mayor complejidad de la estructura tributaria como consecuencia de la aplicación de los gastos tributarios dificulta la fiscalización de los contribuyentes por parte de las autoridades tributarias y generara incentivos a evitar el pago de impuestos.

Este valor de la Eficiencia $\mathrm{C}$ en Argentina puede desagregarse entre las operaciones realizadas en el mercado doméstico y el resultado de importaciones. $\mathrm{La}$ AFIP identifica separadamente la recaudación de IVA impositivo (mercado doméstico) del IVA aduana (proveniente de las importaciones). Si se realiza nuevamente la estimación de la Eficiencia C, separando ambos conceptos, se obtiene que (I) la eficiencia C para el consumo doméstico es del 38\% y (II) para el consumo vinculado al comercio exterior es del $78 \%$. 
Este comportamiento es explicado básicamente por la menor evasión en el IVA recaudado en los productos importados (las dificultades para evadir el IVA en aduana son mayores), a diferencia de lo que ocurre en el mercado doméstico.

Tabla 8. Eficiencia "C" en países seleccionados de la OECD Año 2018

\begin{tabular}{lcccc} 
País & $\begin{array}{c}\text { Tasa general } \\
\text { IVA }\end{array}$ & Eficiencia C & $\begin{array}{c}\text { Gasto } \\
\text { tributario }\end{array}$ & Evasión \\
\hline Australia & 10 & 50 & s. i. & s. i. \\
Bélgica & 21 & 48 & 41 & 11 \\
\hline Canadá & 5 & 47 & s. i. & s. i. \\
\hline Dinamarca & 25 & 60 & 36 & 4 \\
\hline Finlandia & 24 & 54 & 40 & 6 \\
\hline Francia & 20 & 49 & 44 & 7 \\
\hline Italia & 22 & 38 & 42 & 20 \\
\hline Japón & 8 & 71 & s. i. & s. i. \\
\hline Gran Bretaña & 20 & 44 & 40 & 16 \\
\hline Nueva Zelanda & 15 & 95 & s. i. & s. i. \\
\hline Chile & 19 & 64 & s. i. & s. i. \\
\hline México & 16 & 33 & s. i. & s. i. \\
\hline Promedio & 19.1 & 56 & & \\
OECD & 15 & & & \\
\hline
\end{tabular}

Fuente: elaboración propia en base a OECD (2019) y Keen (2013). 


\section{CONSIDERACIONES FINALES}

El impuesto al valor agregado se ha constituido a lo largo del tiempo en un tributo clave de la estructura impositiva argentina, con una recaudación de $7.6 \%$ del PBI en el año 2018 y una participación en el total de recursos tributarios del 32.6\%. La alícuota se fue incrementando a lo largo del tiempo hasta llegar al $21 \%$ y la base imponible se ha ensanchado (por ejemplo con la inclusión en la última reforma de los servicios digitales). Sin embargo, existe un conjunto de tratamientos especiales como bienes exentos, alícuotas reducidas (y acrecentadas) y bienes a tasa cero que han alejado el tributo de un diseño ideal basado en la neutralidad del impuesto, que minimice la pérdida de bienestar y maximice la recaudación tributaria.

Existe un amplio consenso en la literatura acerca de que resulta más apropiado un IVA con base imponible amplia, es decir, sin exenciones y con tratamientos uniformes para todos los bienes, incluso aquellos con características de bienes públicos. A su vez, la literatura concuerda en que potenciales inconvenientes distributivos asociados al IVA deberían compensarse con esquemas de transferencias directas y no introduciendo tratamientos especiales en el impuesto.

El elevado gasto tributario y evasión del impuesto al valor agregado muestran que el potencial recaudatorio del IVA es enorme y que cualquier posible reforma tributaria debería poner el foco en la mejora del diseño e implementación de este impuesto.

\section{REFERENCIAS BIBLIOGRÁFICAS}

Abramovsky, L., Phillips, D., \& Warwick, R. (2017). Redistribution, efficiency and the design of VAT: A review of the theory and literature. (IFS Briefing Note No. 212) Recuperado de https://www.ifs.org.uk/uploads/publications/ bns/BN212.pdf

Adam, S., Phillips, D., Smith, S., Bettendorf, L., Boeters, S., Kox, H., \& Giannini, S. (2011). A Retrospective Evaluation of Elements of the EU VAT System: Final Report. TAXUD/2010/DE/328, FWC No. TAXUD/2010/CC/104. Institute for Fiscal Studies. Recuperado de https://ec.europa.eu/taxation customs/sites/taxation/files/docs/body/report_evaluation_vat.pdf

Ahmad, E. \& Best, M. (2012). Financing Social Policy in the Presence of Informality (LSE Asia Research Centre. Working Paper, No. 54). Recuperado de http://eprints.lse.ac.uk/57964/1/_lse.ac.uk_storage_LIBRARY_Second- 
ary_libfile_shared_repository_Content_Asia\%20Research $\% 20$ Centre $\% 20$ Working\%20Papers_ARCWP54-AhmadBest.pdf

Anton, A., Hernandez, F. \& Levy, S. (2013). The End of Informality in Mexico? Fiscal Reform for Universal Social Insurance. Washington, D.C.: Inter-American Development Bank. Recuperado de https://publications. iadb.org/publications/english/document/The-End-of-Informality-in-Mexico-Fiscal-Reform-for-Universal-Social-Insurance.pdf

Agha, A. \& Haughton, J. (1996). Designing Vat Systems: Some Efficiency Considerations. Review of Economics and Statistics, 78(2), 303-308.

Annacondia, F. (2019). International - Overview of General Turnover Taxes and Tax Rates - January 2019. International VAT Monitor, 30(2).

Artana, D., Guardarucci, I., Lavigne, P., Puig, J. \& Susmel, N. (2015). El sistema tributario argentino. Análisis y evaluación de propuestas para reformarlo. (Documento de Trabajo FIEL N¹23). Buenos Aires: Fundación de Investigaciones Económicas Latinoamericanas

Aujean, M., Jenkins, P., \& Poddar, S. (1999). A New Approach to Public Sector Bodies. International VAT Monitor, 10(4), 144-149.

Barbone, L., Bird, R., \& Vazquez-Caro, J. (2012). The Costs of VAT: A Review of the Literature. SSRN Electronic Journal. (International Center for Public Policy Working Paper 12-22). Recuperado de https://icepp.gsu.edu/ files/2015/03/ispwp1222.pdf

Bird, R. (2011). Commentary on Crawford, Keen and Smith. In J. Mirrlees, S. Adam, T. Besley, R. Blundell, S. Bond, R. Chote, M. Gammie, P. Johnson, G. Myles \& J. Poterba (Eds). Dimensions of Tax Design. (Cap. 4: Value Added Tax and Excises, pp. 363-369). New York: Oxford University Press. Recuperado de https://www.ifs.org.uk/uploads/mirrleesreview/dimensions/ ch4.pdf

Bird, R., \& Gendron, P. (2007). The VAT in Developing and Transitional Countries. Cambridge: Cambridge University Press.

Bird, R., Gendron, P., \& Rotman, L. (2005). VAT Revisited A New Look at the Value Added Tax in Developing and Transitional Countries. Toronto: USAID.

Bogetic, Z., \& Hassan, F. (1993). Determinants of Value-Added Tax Revenue: A Cross-section Analysis. (Policy Research Working Paper Series No. 1203). Washington, D.C.: The World Bank.

Buettner, T., \& Erbe, K. (2014). Revenue and welfare effects of financial sector VAT exemption. International Tax and Public Finance, 21(6), 1028-1050.

Cnossen, S. (2004), VAT in South Africa: what kind of rate structure? International VAT Monitor 15, 19-24 
Comisión Económica para América Latina y el Caribe (2019). Panorama Fiscal de América Latina y el Caribe 2019. Recuperado de https://www.cepal.org/es/ publicaciones/44516-panorama-fiscal-america-latina-caribe-2019-politicastributarias-la-movilizacion

Copenhagen Economics \& KPMG (2011). VAT in the Public Sector and Exemptions in the Public Interest. Recuperado de https://ec.europa.eu/taxation customs/sites/taxation/files/resources/documents/common/publications/ studies/vat_public_sector_en.pdf

de la Feria, R., \& Krever, R. (2013). Ending VAT Exemptions: Towards a Post-Modern VAT. In R. de la Feria (Ed.), VAT Exemptions: Consequences and Design Alternatives (Cap. 1, pp. 3-36). The Netherlands: Wolters Kluwer.

Dickson, I. D., \& White, D. (2011). Commentary on Crawford, Keen and Smith. In Mirrlees J., S. Adam, T. Besley, R. Blundell, S. Bond, R. Chote, M. Gammie, P. Johnson, G. Myles and J. Poterba (Eds). Dimensions of Tax Design. The Mirrlees Report. New York: Oxford University Press.

Ebrill, L., Keen, M., Bodin, J., \& Summers, V. (2001) The Modern VAT. Washington D. C.: International Monetary Fund.

Ebrill, L., Keen, M., Bodin, J., \& Summers, V. (2002). The allure of the value-added tax. Finance and Development 39(2), 44-47.

Ernst \& Young (2019). Worldwide VAT, GST and Sales Tax Guide. Recuperado de https://taxnews.ey.com/news/2019-1031-eys-worldwide-vat-gst-and-salestax-guide-2019?uAlertID=Sd\%2fG8rua1oj6\%2fl58EZ2AiA\%3d\%3d

Fernández Felices, D., Guardarucci, I., \& Puig, J. (2017). El Impuesto al Valor Agregado en Argentina. Consideraciones sobre su incidencia distributiva y propuestas de reforma. Actualidad Económica, 26(90), 29-38. Recuperado de https://revistas.unc.edu.ar/index.php/acteconomica/article/ view/16744/16521

Fundación de Investigaciones Económicas Latinoamericanas (1998). La Reforma Tributaria en la Argentina. Buenos Aires: FIEL. Recuperado de http:// www2.congreso.gob.pe/sicr/cendocbib/con4_uibd.nsf/F641869E11B6B2 A205257FD200778E9F/\$FILE/reformatrib.pdf

Gasparini, L. (1998). Incidencia distributiva del sistema impositivo argentino. En La reforma tributaria en la Argentina. Buenos Aires: FIEL.

Gauthier, B., \& Gersovitz, M. (1997). Revenue Erosion through Exemption and Erosion in Cameroon, 1993. Journal of Public Economics, 64(3), 407-424 Gendron, P. (2005). Value-Added Tax Treatment of Public Bodies and NonProfit Organizations: A Developing Country Perspective Bulletin for International Fiscal Documentation, 59, 514-525. 
Gómez Sabaini, J. C., Jiménez, J. P. y Martner, R. (Eds.) (2017). Consensos y conflictos en la política tributaria de América Latina. Santiago: CEPAL. Recuperado de https://repositorio.cepal.org/bitstream/handle/11362/41048/6/ S1700003_es.pdf

Gómez Sabaini, J. C., \& Morán, D. (2014). Tax policy in Latin America. (Series Macroeconomics of Development No. 133). Recuperado de http://www. obela.org/system/files/TaxpolicyinLatinAmerica_CEPAL.pdf

Harris, T., Phillips, D., Warwick, R., Goldman, M., Jellema, J., Goraus, K., \& Inchauste, G. (2018). Redistribution via VAT and cash transfers: an assessment in four low and middle income countries (Institute for Fiscal Studies. No. W18/11).

Huizinga, H. (2002). A European VAT on financial services? Economic Policy, 17(35), 497-534.

International Monetary Fund (2010). Fiscal Exit: From Strategy to Implementation. In Fiscal Monitor. Recuperado de https://www.elibrary.imf.org/doc/ IMF089/11288-9781616350475/11288-9781616350475/Other_formats/ Source_PDF/11288-9781616350499.pdf

Jack, W. (1996). The efficiency of VAT implementation: a comparative study of Central and Eastern European countries in transition. (Working Paper No. 96/79). Washington, D.C.: International Monetary Fund.

James, K. (2015). Introduction. In The Rise of the Value-Added Tax. Cambridge: Cambridge University Press.

Keen M., \& Lockwood, B. (2006). Is the VAT a Money Machine? National Tax Journal, 59(4), 905-928.

Keen, M., \& Smith, S. (2006). VAT Fraud and Evasion: What Do We Know and What Can Be Done? National Tax Journal, 59(4), 861-887.

Keen, M. (2013). The Anatomy of the VAT. (IMF Working Paper No. 13/111). Recuperado de https://www.imf.org/external/pubs/ft/wp/2013/wp13111.pdf

Mirrlees, J. (2011). Tax by design: The Mirrlees Review. Oxford: Institute for Fiscal Studies.

OECD (2018) Consumption Tax Trends 2018: VAT/GST and Excise Rates, Trends and Policy Issues, Recuperado de https://doi.org/10.1787/ctt-2018-en.

Poddar, S. (2005). Application of VAT to Public Bodies. Presented at the VAT Conference, International Tax Dialogue, Rome, March 15-16.

Reckon LLP (2009). Study to quantify and analyse the VAT gap in the EU-25 Member States. Taxation Studies 0029, Directorate General Taxation and Customs Union, European Commission. Recuperado de https://ec.europa.eu/ taxation_customs/sites/taxation/files/docs/body/reckon_report_sep2009.pdf 
Ríos, G. (2009). Informe $N^{o} 13$ Área Tributaria: El impuesto al valor agregado en la actividad bancaria. Recuperado de: https://www.facpce.org.ar/pdf/cecyt/ tributaria-13.pdf

Schwab R. \& Oates W. (2015). The Window Tax: A Case Study in Excess Burden. (Lincoln Institute of Land Policy. Working Paper No. 14RS1). Recuperado de https://www.lincolninst.edu/sites/default/files/pubfiles/2380_1720_ Schwab_WP14RS1.pdf

Slemwd, J. (1989). Complexity, Compliance Costs, and Tax Evasion. In J. Roth \& J. Scholz (Eds.), Taxpayer Compliance, Volume 2: Social Science Perspectives (pp. 156-181). Philadelphia: University of Pennsylvania Press.

Ueda, J. (2017). The Evolution of Potential VAT Revenues and C-Efficiency in Advanced Economies. (IMF Working Paper No. 17/158). Recuperado de https://www.imf.org/en/Publications/WP/Issues/2017/07/11/The-Evolution-of-Potential-VAT-Revenues-and-C-Efficiency-in-Advanced-Economies-45015

Zee, H. (1995). Taxation and Efficiency and Taxtation and Equity. En P. Shome (Ed.) Tax Policy Handbook (pp. 25-34). Washington, D.C.: International Monetary Fund.

(C) 2021 por los autores; licencia no exclusiva otorgada a la revista Estudios económicos. Este artículo es de acceso abierto y distribuido bajo los términos y condiciones de una licencia Atribución-No Comercial 4.0 Internacional (CC BY-NC 4.0) de Creative Commons. Para ver una copia de esta licencia, visite http://creativecommons.org/licenses/by-nc/4.0 